\title{
Dietary intake of flavonoids and oesophageal and gastric cancer: incidence and survival in the United States of America (USA)
}

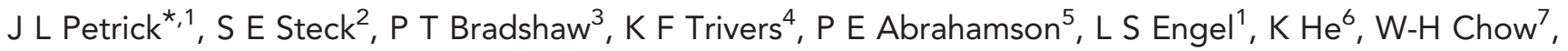 \\ S T Mayne ${ }^{8}$, H A Risch ${ }^{8}$, T L Vaughan ${ }^{9}$ and M D Gammon ${ }^{1}$ \\ ${ }^{1}$ Department of Epidemiology, CB 7435, University of North Carolina, Chapel Hill, NC 27599-7435, USA; ${ }^{2}$ Department of \\ Epidemiology and Biostatistics, University of South Carolina, Columbia, SC, USA; ${ }^{3}$ Department of Nutrition, University of North \\ Carolina, Chapel Hill, NC, USA; ${ }^{4}$ Division of Cancer Prevention and Control, Centers for Disease Control, Atlanta, GA, USA; \\ ${ }^{5}$ Salmon Bay Consulting, Inc., Seattle, WA, USA; ${ }^{6}$ Department of Epidemiology and Biostatistics, Indiana University, Bloomington, \\ IN, USA; ${ }^{7}$ Department of Epidemiology, University of Texas MD Anderson Cancer Center, Houston, TX, USA; ${ }^{8}$ Department of \\ Chronic Disease Epidemiology, Yale School of Public Health and Yale Cancer Center, New Haven, CT, USA and ${ }^{9}$ Division of Public \\ Health Sciences, Fred Hutchinson Cancer Research Center, Seattle, WA, USA
}

Background: Flavonoids, polyphenolic compounds concentrated in fruits and vegetables, have experimentally demonstrated chemopreventive effects against oesophageal and gastric cancer. Few epidemiologic studies have examined flavonoid intake and incidence of these cancers, and none have considered survival.

Methods: In this USA multicentre population-based study, case participants (diagnosed during 1993-1995 with oesophageal adenocarcinoma (OEA, $n=274$ ), gastric cardia adenocarcinoma (GCA, $n=248$ ), oesophageal squamous cell carcinoma (OES, $n=191)$, and other gastric adenocarcinoma (OGA, $n=341)$ ) and frequency-matched controls $(n=662)$ were interviewed. Food frequency questionnaire responses were linked with USDA Flavonoid Databases and available literature for six flavonoid classes and lignans. Case participants were followed until 2000 for vital status. Multivariable-adjusted odds ratios (ORs) and hazard ratios (HRs) (95\% confidence intervals (Cls)) were estimated, comparing highest with lowest intake quartiles, using polytomous logistic and proportional hazards regressions, respectively.

Results: Little or no consistent association was found for total flavonoid intake (main population sources: black tea, orange/ grapefruit juice, and wine) and incidence or survival for any tumour type. Intake of anthocyanidins, common in wine and fruit juice, was associated with a 57\% reduction in the risk of incident OEA $(\mathrm{OR}=0.43,95 \% \mathrm{Cl}=0.29-0.66)$ and $\mathrm{OES}(\mathrm{OR}=0.43,95 \%$ $\mathrm{Cl}=0.26-0.70)$. The ORs for isoflavones, for which coffee was the main source, were increased for all tumours, except OES. Anthocyanidins were associated with decreased risk of mortality for $\mathrm{GCA}(\mathrm{HR}=0.63,95 \% \mathrm{Cl}=0.42-0.95)$ and modestly for OEA (HR $=0.87,95 \% \mathrm{Cl}=0.60-1.26)$, but $\mathrm{Cls}$ were wide.

Conclusions: Our findings, if confirmed, suggest that increased dietary anthocyanidin intake may reduce incidence and improve survival for these cancers.

Over the past two decades, oesophageal adenocarcinoma (OEA) and gastric cardia adenocarcinoma (GCA) have been among the most rapidly increasing cancer types in the United States and other
Western countries (Devesa et al, 1998; Simard et al, 2012). Oesophageal adenocarcinoma and GCA are often considered as one clinical entity because they are both epithelial cancers

*Correspondence: Dr JL Petrick; E-mail: jessica.petrick@unc.edu

Received 7 August 2014; revised 16 December 2014; accepted 8 January 2015; published online 10 February 2015

(c) 2015 Cancer Research UK. All rights reserved 0007-0920/15 
originating in or near the gastroesophageal junction and have similar 5-year survival rates of $\sim 26 \%$ (Wijnhoven et al, 1999). Still, oesophageal squamous cell carcinoma (OES) and other (noncardia) gastric adenocarcinoma (OGA) are the most common forms of oesophageal and gastric cancer worldwide. In addition to geographic variation, these four cancer types have differing risk factors (Hongo et al, 2009; de Martel et al, 2013). Thus, considering them as distinct entities will help to elucidate the underlying aetiology.

Epidemiologic studies have shown that diets high in fruits and vegetables are inversely associated with oesophageal and gastric cancer incidence (Lunet et al, 2007; Li et al, 2014). Flavonoids, which are a group of bioactive polyphenolic compounds naturally occurring in fruits, vegetables, and beverages of plant origin, have been hypothesised to account at least partially for such risk reductions (Pierini et al, 2008). Experimental studies have supported this hypothesis. For example, freeze-dried berries, which are high in anthocyanidins, inhibited $24-56 \%$ of oesophageal tumour multiplicity (average number of tumours per oesophagus) and $8-21 \%$ of incidence in rats caused by $N$-nitrosomethylbenzylamine compared with controls (Stoner et al, 2006). In addition, Flavopiridol, a synthetic flavone, has been studied in small phase I and II clinical trials in patients with metastatic gastric cancer but was an inactive chemopreventive strategy (Schwartz et al, 2001). However, this clinical trial was undertaken without any epidemiologic evidence to support the use of flavonoids. Lignans are another group of polyphenolic compounds that have anti-inflammatory and pro-apoptotic effects, antioxidant properties, and promote cell cycle arrest (Huang et al, 2010).

Recent epidemiological investigations conducted in the United States (Bobe et al, 2009), Greece (Lagiou et al, 2004), Italy (Rossi et al, 2007, 2010), Europe (Zamora-Ros et al, 2012; Vermeulen et al, 2013), Sweden (Ekstrom et al, 2011), China (Sun et al, 2002), and Mexico (Hernandez-Ramirez et al, 2009) have analysed associations between flavonoid or lignan intake and oesophageal or gastric cancer incidence. However, no epidemiologic studies to date have examined associations between flavonoid intake and survival among oesophageal or gastric cancer patients. Clarification of whether total flavonoids, or specific flavonoid classes, influence the incidence of these tumour subtypes or survival once diagnosed would provide empirical support for developing potential risk reduction strategies utilising these compounds.

In this population-based study, we investigated whether intakes of total flavonoids, including specific flavonoid classes, and lignans are associated with: (1) oesophageal or gastric cancer incidence and (2) survival among individuals diagnosed with oesophageal or gastric cancer. For both aims, we consider associations for all four cancer types separately.

\section{MATERIALS AND METHODS}

To conduct this ancillary study, we built upon the resources from the USA Multicenter Study, a population-based investigation conducted in the state of Connecticut (CT, principal investigator (PI): HA Risch), a 15-county area of New Jersey (NJ, PI: MD Gammon), and a three-county area of western Washington state (WA, PI: TL Vaughan), which was initiated as a case-control study (Gammon et al, 1997) and then continued as a follow-up study to determine vital status among the cases (Trivers et al, 2005). This study was approved by all Institutional Review Boards of the participating institutions.

Study population. The three geographic areas (CT, NJ, and WA) each had a population-based cancer registry that was used to identify the cases through rapid reporting methods. Eligible case participants were English-speaking men and women between the ages of 30 and 79 years, diagnosed with a first primary invasive oesophageal or stomach cancer between 1993 and 1995. The parent study goal was to recruit all individuals newly diagnosed with OEA and GCA (which were considered the target case participants), whereas participants with OES or OGA (i.e., comparison cases) were frequency matched to the expected target case participants by geographic location and 5-year age group (CT, NJ, and WA), sex (NJ and WA), and race (white or other, NJ). Final determination of case participant eligibility and classification was made by study pathologists (Drs Heidi Rotterdam at Columbia University for NJ and A Brian West at New York University for CT and WA) after review of medical records and pathology specimens (Gammon et al, 1997).

Population-based control participants were frequency matched to the target case participants by 5-year age group and sex. Control participants 30-64 years of age were identified using a modified Waksberg random digit dialing technique (Waksberg, 1978). Control participants 65-79 years of age were identified by random sampling of Health Care Financing Administration rosters (now Centers for Medicare and Medicaid Services) (Gammon et al, 1997).

Study participants included 293 individuals with OEA and 261 with GCA ( $80.6 \%$ of eligible target case participants), 221 with OES and 368 with OGA $(74.1 \%$ of eligible comparison case participants), and 695 population control participants (74.1\% of eligible control participants) (Gammon et al, 1997). The $93.4 \%$ of participants with dietary intake information are the focus of the current study, and the distribution of the demographic characteristics of this subsample did not differ substantially from those of all study participants (Mayne et al, 2001) (Supplementary Table 1). Males comprised $83.9 \%$ of target cases, $72.9 \%$ of comparison cases, and $79.8 \%$ of controls; $98.1 \%$ of target cases, $83.3 \%$ of comparison cases, and $93.2 \%$ of controls were white; and $78.4 \%$ of target cases, $66.0 \%$ of comparison cases, and $79.2 \%$ of controls had more than a high school education.

Exposure assessment. Information on demographic factors, tobacco and alcohol use, intake of other beverages (e.g., coffee and tea), medical history, medication use, and occupational history was obtained by a structured questionnaire administered face to face by trained interviewers. Because of the high lethality of these tumours, proxy interviews (usually with a spouse or other next-ofkin) were required for $29.6 \%$ of target case participants, $32.2 \%$ of comparison case participants, and $3.4 \%$ of control participants. We analysed the data both including and excluding proxy interviews and the results were consistent (Supplementary Tables 2 and 3), and therefore we present the analysis including the proxy data. The average time between cancer diagnosis and interview for cases was 3.7 months for self-report and 8.5 months for proxy report. Interviews averaged $130 \mathrm{~min}$. Written informed consent was obtained from each participant before the interview (Gammon et al, 1997).

Dietary data were collected by interviewers using a 104-item food frequency questionnaire (FFQ) modified from one developed and validated by investigators at the Fred Hutchinson Cancer Research Center (FHCRC) (Kristal et al, 1997). Participants were asked to report usual dietary intake for 3-5 years before diagnosis (case participants) or interview (control participants). Completed FFQs were not obtained for 33 participants who provided only abbreviated interviews. Estimated total energy intake of $<500$ or $>4000$ kilocalories per day for women and $<800$ or $>5000$ kilocalories per day for men were considered implausible intake values, thereby excluding 61 case and 28 control participants (Willett, 2012). Final numbers of participants included in this study were 274 with OEA, 248 with GCA, 191 with OES, and 341 with OGA cases, and 662 control participants.

Assessment of dietary flavonoid intake. A study-specific flavonoid database was created based on values from the 
2011 United States Department of Agriculture (USDA) Database for the Flavonoid Content of Selected Foods (Bhagwat et al, 2011) and the 2008 USDA-Iowa State University Database on the Isoflavone Content of Selected Foods (Bhagwat et al, 2008) and supplemented with lignan content data (i.e., secoisolariciresinol and matairesinol) (Thompson et al, 2006). Using FFQ reported frequencies of dietary intakes, intake of total flavonoids and six classes of flavonoids (anthocyanidins, flavan-3-ols, flavanones, flavones, flavonols, and isoflavones) and lignans were estimated using the study-specific flavonoid database for 81 food and beverage items that contained measureable amounts of flavonoids (Supplementary Table 4).

When FFQ items represented groups of foods or beverages, the individual foods and beverages were weighted based on usual population consumption. For example, the FFQ item of 'apples and pears' was assigned weights of 0.75 for 'apples' and 0.25 for 'pears'. Flavonoid intake was calculated by multiplying the weight assigned to each food in the FFQ item by the flavonoid content of that food, summing across all foods in the FFQ item, and then multiplying by the number of times consumed per day. For example, $100 \mathrm{~g}$ of apple contains $1.29 \mathrm{mg}$ and pears contain $12.18 \mathrm{mg}$ of anthocyanidins. A serving size of apples or pears was estimated as $145 \mathrm{~g}$. Therefore, if an individual reported consuming one serving of apples or pears per day, the individual's daily intake of anthocyanidins from apples and pears was calculated as $145 \mathrm{~g}$ apple or pear per day $\times[(0.75$ apple weight $\times 1.29 \mathrm{mg}$ per $100 \mathrm{~g}$ apples $)+(0.25$ pear weight $\times 12.18 \mathrm{mg}$ per $100 \mathrm{~g}$ pear $)]=5.82 \mathrm{mg}$ anthocyanidins per day.

Outcome assessment. In follow-up of the Multicenter Study, vital status and date of death were determined by linking state tumour registry data with the National Death Index. Overall survival time (in months) was calculated from the date of diagnosis until death or last follow-up, with a maximum follow-up of 90 months, ending in 2000. Median survival time was 9.6 months for the study participants diagnosed with OEA, 12.8 months for those with GCA, 10.7 months for OES, and 12.9 months for OGA. At the end of follow-up, participants who were still alive were considered censored. The outcome was death from any cause (Trivers et al, 2005).

Statistical analysis. All analyses were conducted using SAS version 9.2 (SAS Institute, Cary, NC, USA).

Case-control analysis. Polytomous unconditional logistic regression was used to calculate odds ratios (ORs) and their 95\% confidence intervals (CIs) for the association between flavonoid/ lignan intake and the risk of incidence for the four tumour types (OEA, GCA, OES, and OGA) in comparison with control participants. Effect measure modification by cigarette smoking (evaluated as ever/never and continuous duration), usual adult body mass index (BMI, evaluated as continuous and as dichotomised, $<25$ and $\geqslant 25 \mathrm{~kg} \mathrm{~m}^{-2}$ ), and self-reported gastroesophageal reflux disease (yes/no) was assessed using likelihood ratio statistics to compare regression models with and without a multiplicative term (Rothman et al, 2008). There was no evidence for effect modification by any of these variables $(P \geqslant 0.05)$ on the association between flavonoid intake and oesophageal or gastric cancer.

Potential confounders for the case-control analysis included proxy status (proxy, non-proxy), income (evaluated as $<\$ 15000$, \$15000-29 999, \$30 000-49 999, \$50 000-74 999, or $\geqslant \$ 75000$ and $<\$ 15000$ or $\geqslant \$ 15000$ ), education (evaluated as $<$ high school, high school, technical school/some college, or $\geqslant$ college and $<$ high school or $\geqslant$ high school), cigarette smoking (evaluated as ever/ never, continuous pack-years, and continuous cigarettes/day), alcohol consumption (evaluated as ever/never and continuous for beer, wine, and liquor), and BMI (evaluated as continuous and categorised, $<25,25-29.9$, or $\geqslant 30 \mathrm{~kg} \mathrm{~m}^{-2}$ ). If the log OR changed by $\geqslant 10 \%$ because of variable elimination, the variable was considered a confounder and remained in the model (Rothman et al, 2008); only cigarette smoking met this criterion. Total energy intake was included for adjustment as an a priori confounder (Willett et al, 1997). Thus, all final logistic regression models include cigarette smoking (ever/never), kilocalories (continuous), and the frequency matching factors of site (CT, NJ, and WA), age (continuous), sex (male, female), and race (white, other). Although we chose to present results adjusted utilising the standard multivariate approach for ease of interpretation, we also examined utilising the nutrient density and residual models and results did not differ (Willett, 2012) (Supplementary Table 5). Ratios of the ORs were calculated to determine heterogeneity between OEA and the other cancer subtypes.

Survival analysis. Cox proportional hazard regression analysis was used to calculate adjusted hazard ratios (HRs) and their 95\% CIs for the association between flavonoid/lignan intake and mortality for each tumour type as distinct outcomes. The proportional hazards assumption was tested utilising an interaction with $\log$ (time) in models with confounders, and it was not observed to be violated.

Potential confounders for the survival analysis included the same covariates listed above in the case-control analysis, as well as study site (WA, NJ, and CT), age (continuous), sex (male, female), tumour stage (localised, regional, distant, and unknown), tumour grade (well/moderate, poor/undifferentiated, and not determined), and dysphagia (yes, no). Variables remained in the adjusted model if they were significant predictors of survival $(P<0.05)$ (Rothman et al, 2008); only stage met this criterion. Total energy intake was included as an a priori confounder (Willett et al, 1997). Thus, all final proportional hazard models included tumour stage and kilocalories (continuous). Stratified Cox proportional hazard regression analysis, with interaction terms between cancer type and flavonoids, tumour stage, and total energy intake, was used to calculate the ratio of the HRs to determine heterogeneity between EA and the other cancer subtypes.

Examination of linear trend. Flavonoid intake was categorised as quartiles, based on the distribution of intake among the controls (logistic regression) or all cases (survival analysis) (Willett, 2012). To examine trends, we evaluated flavonoid intake with restricted quadratic splines as well as linear trends in continuous flavonoid values (mg per day).

Sensitivity analysis. For anthocyanidins, a value of $7.39 \mathrm{mg}$ per $100 \mathrm{~g}$ of banana is assigned in the USDA Flavonoid Database (Bhagwat et al, 2011). However, this value is controversial (Drossard et al, 2011). Thus, we conducted sensitivity analyses that excluded this value (Supplementary Tables 6-9). We also stratified incidence and mortality risk estimates by tumour stage (Supplementary Tables 10 and 11).

\section{RESULTS}

As shown in Table 1, control participants consumed on average similar amounts of total flavonoids (median $=97.09 \mathrm{mg}$ per day) as case participants (OEA median $=96.89$; GCA median $=104.27$; OES median $=108.97$; OGA median $=101.32 \mathrm{mg}$ per day). Flavan3-ols were the largest contributor to total flavonoid intake in this study population, and control participants consumed less flavan3-ols than OEA, GCA, and OGA case participants.

Table 2 lists the major sources of flavonoids and lignans in the reported food items of the control participants. For total flavonoids, black tea provided $55.8 \%$ ( $105.11 \mathrm{mg}$ per day) of mean intake, orange/grapefruit juice $14.2 \%$ (26.75 mg per day), and wine 


\begin{tabular}{|c|c|c|c|c|c|c|c|c|c|}
\hline 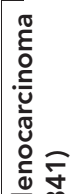 & 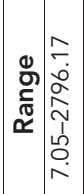 & \begin{tabular}{l}
$\infty$ \\
0 \\
$o$ \\
0 \\
1 \\
\multirow{2}{*}{} \\
\multirow{2}{0}{} \\
0
\end{tabular} & 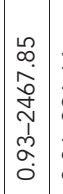 & 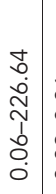 & $\begin{array}{l} \\
\delta \\
0 \\
1 \\
\tilde{1} \\
0 \\
0\end{array}$ & 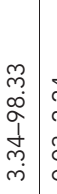 & 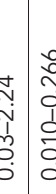 & 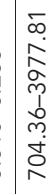 & \\
\hline 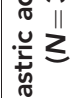 & 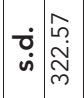 & $\stackrel{\infty}{\stackrel{\infty}{a}}$ & 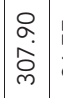 & 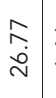 & 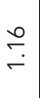 & 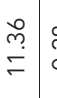 & 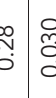 & $\left\{\begin{array}{l}\sim \\
\infty \\
\infty \\
\infty \\
\infty \\
\infty\end{array}\right.$ & \\
\hline \begin{tabular}{l}
$\frac{1}{\Phi}$ \\
\multirow{0}{0}{}
\end{tabular} & 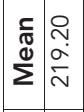 & 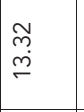 & 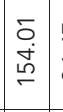 & $\begin{array}{l}\hat{f} \\
\dot{m}\end{array}$ & $\stackrel{\tilde{N}}{\stackrel{N}{S}}$ & $\begin{array}{l}\stackrel{\partial}{\alpha} \\
\dot{j} \\
\dot{f}\end{array}$ & 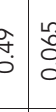 & 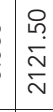 & \\
\hline 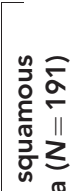 & 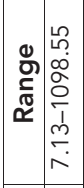 & 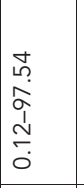 & 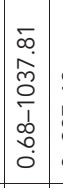 & 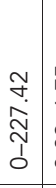 & 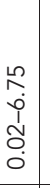 & 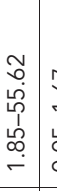 & 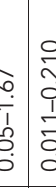 & 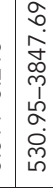 & \\
\hline 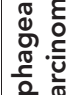 & 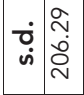 & 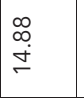 & 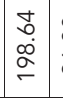 & $\begin{array}{l}\infty \\
\stackrel{\infty}{0} \\
\stackrel{\leftrightarrow}{\sim}\end{array}$ & $\stackrel{\stackrel{\overbrace{}}{ }}{\longrightarrow}$ & 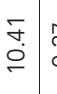 & 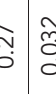 & 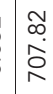 & \\
\hline Oัँ $\overline{\bar{\Phi}}$ & 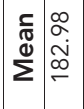 & $\underset{i n}{i n}$ & 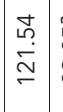 & 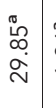 & 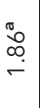 & $\begin{array}{l}\stackrel{+}{\stackrel{f}{\rho}} \\
\stackrel{\rho}{\rho}\end{array}$ & 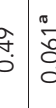 & 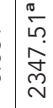 & \\
\hline 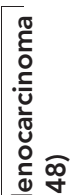 & 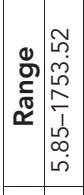 & 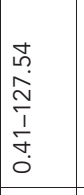 & \begin{tabular}{|c|}
0 \\
0 \\
0 \\
0 \\
0 \\
1 \\
0 \\
0 \\
0 \\
0 \\
0
\end{tabular} & 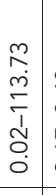 & $\begin{array}{c}0 \\
q \\
0 \\
1 \\
1 \\
\sigma \\
0\end{array}$ & 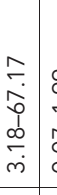 & 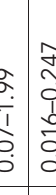 & $\mid \begin{array}{l}0 \\
0 \\
0 \\
0 \\
0 \\
0 \\
1 \\
0 \\
0 \\
0 \\
i\end{array}$ & \\
\hline 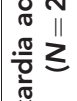 & 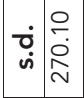 & $\begin{array}{l}\bar{m} \\
\stackrel{\infty}{\infty}\end{array}$ & 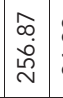 & $\begin{array}{l}\sigma \\
\sigma \\
\dot{v}\end{array}$ & $\stackrel{\stackrel{a}{p}}{\sim}$ & $\begin{array}{l}m \\
\stackrel{3}{0} \\
\stackrel{2}{0}\end{array}$ & $\begin{array}{c}\tilde{m} \\
\delta \\
\delta\end{array}$ & $\mid \begin{array}{l}0 \\
0 \\
0 \\
0 \\
0\end{array}$ & \\
\hline 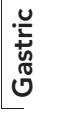 & 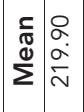 & 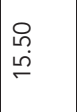 & 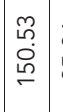 & $\begin{array}{l}\text { ț } \\
\dot{m}\end{array}$ & $\stackrel{\substack{* \\
\sim}}{\sim}$ & 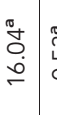 & 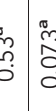 & 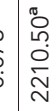 & \\
\hline 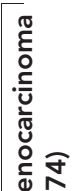 & 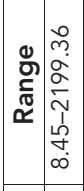 & $\begin{array}{l}0 \\
0 \\
0 \\
0 \\
00 \\
1 \\
0 \\
0 \\
0 \\
0\end{array}$ & 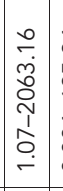 & 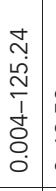 & \begin{tabular}{c|}
$\stackrel{0}{0}$ \\
$\stackrel{0}{+}$ \\
$\stackrel{1}{0}$
\end{tabular} & 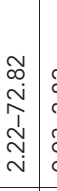 & 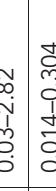 & 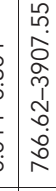 & \\
\hline 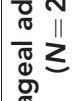 & : & \begin{tabular}{l}
0 \\
0 \\
0 \\
\hdashline
\end{tabular} & $\mid \begin{array}{c}m \\
m \\
\dot{p} \\
\sim \\
\sim\end{array}$ & 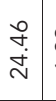 & 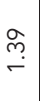 & 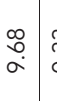 & $\begin{array}{l}m \\
\text { ô } \\
\end{array}$ & 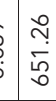 & \\
\hline 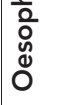 & 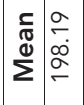 & 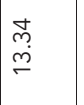 & 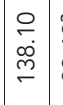 & \begin{tabular}{l}
$\stackrel{\pi}{\sim}$ \\
\multirow{\sim}{\sim}{} \\
$\sim$
\end{tabular} & $\stackrel{a}{\stackrel{i}{~}}$ & \begin{tabular}{l|l}
$\stackrel{P}{r}$ & \\
$\dot{+}$ &
\end{tabular} & $\begin{array}{lll}0 \\
0 \\
0\end{array}$ & : & \\
\hline$\frac{4}{0} \widetilde{7}$ & 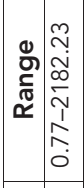 & $\begin{array}{c}\infty \\
\infty \\
\infty \\
\infty \\
0 \\
0\end{array}$ & 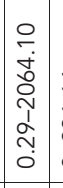 & 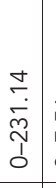 & 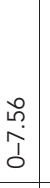 & \begin{tabular}{c|c}
0 & 0 \\
0 & 0 \\
0 & 0 \\
1 & 0 \\
0 & 0 \\
0 & $\vdots$ \\
0
\end{tabular} & 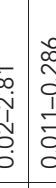 & 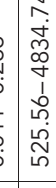 & 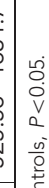 \\
\hline$\dot{u}^{\circ} \geq$ & 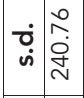 & 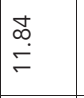 & 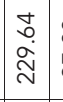 & $\begin{array}{l}8 \\
\stackrel{a}{N}\end{array}$ & 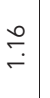 & $\begin{array}{l}\check{\sigma} \\
\sigma\end{array}$ & 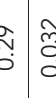 & 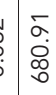 & 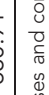 \\
\hline & 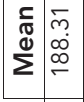 & $\begin{array}{l}\infty \\
\stackrel{\infty}{\sim} \\
\stackrel{+}{+}\end{array}$ & $\mid \begin{array}{l}\bar{\sigma} \\
\dot{\bar{N}} \\
\end{array}$ & $\begin{array}{l}m \\
\alpha \\
\dot{m} \\
\dot{m}\end{array}$ & $\stackrel{\text { ì }}{\text { in }}$ & 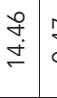 & $\begin{array}{ll}f \\
0\end{array}$ & 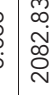 & 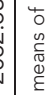 \\
\hline & 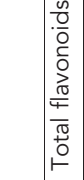 & 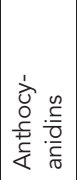 & 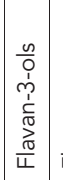 & $\begin{array}{l}0 \\
0 \\
0 \\
0 \\
\frac{0}{0} \\
\frac{w}{W L} \\
\end{array}$ & 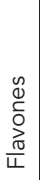 & $\begin{array}{c}\frac{0}{0} \\
0 \\
0 \\
0 \\
\frac{0}{L} \\
\end{array}$ & 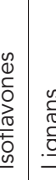 & 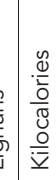 & 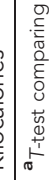 \\
\hline
\end{tabular}

$4.5 \%$ (8.46 mg per day). Black tea was a source of flavan-3-ols, flavonols, and lignans. Orange/grapefruit juice provided flavanones, flavonols, isoflavones, and lignans. Wine was a source of anthocyanidins, flavan-3-ols, flavanones, flavones, flavonols, and lignans.

As shown in Table 3, intake of dietary anthocyanidins, for which wine, bananas, and fruit juice were the major dietary sources, was inversely associated with risk of incidence for all tumour types, although the magnitude and precision of the estimates varied by tumour type. Comparing the highest with lowest quartile of anthocyanidin intake, decreased risks were shown for incident OEA $\left(\mathrm{OR}=0.43,95 \% \mathrm{CI}=0.29-0.66, P_{\text {trend }}=0.06\right)$ and $\mathrm{OES}$ $\left(\mathrm{OR}=0.43,95 \% \mathrm{CI}=0.26-0.70, \quad P_{\text {trend }}=0.11\right)$. More modest decreased risks were shown for incident GCA (OR $=0.71,95 \%$ $\mathrm{CI}=0.46-1.10)$ and $\mathrm{OGA}(\mathrm{OR}=0.70,95 \% \mathrm{CI}=0.47-1.03)$, but the $\mathrm{CI}$ included the null value.

For all other flavonoid types, the associations with incident oesophageal and gastric cancers were less consistent. For example, as also shown in Table 3, dietary flavanone intake was inversely associated with incident $\mathrm{OEA}(\mathrm{OR}=0.56,95 \% \mathrm{CI}=0.37-0.85$, $\left.P_{\text {trend }}=0.003\right) \quad$ and $\mathrm{OES} \quad(\mathrm{OR}=0.48,95 \% \mathrm{CI}=0.29-0.78$, $\left.P_{\text {trend }}=0.002\right)$. Dietary flavone and lignan intake were inversely associated with $\mathrm{OES}$ incidence $(\mathrm{OR}=0.55,95 \% \mathrm{CI}=0.34-0.89$, $P_{\text {trend }}=0.02 ; \quad \mathrm{OR}=0.38,95 \% \quad \mathrm{CI}=0.23-0.63, \quad P_{\text {trend }}=0.0003$, respectively). Lignan intake was more modestly associated with incident OEA ( $\mathrm{OR}=0.75,95 \% \mathrm{CI}=0.49-1.13)$, but the CI included the null value.

As also shown in Table 3, we observed a modest inverse association between dietary intake of isoflavones, for which coffee, chili, and white bread were the major dietary sources, and OES incidence, but the CI included the null value; in contrast, a positive association was observed for isoflavones in relation to all other tumour types. Similarly, we observed a modest positive association for dietary flavonol intake with GCA incidence, but not other tumour types. Little or no consistent association was observed between total flavonoid intake or flavan-3-ols, accounting for $64.6 \%$ of total flavonoid intake among controls, and any tumour types.

As presented in Table 4, anthocyanidin intake was associated with a decreased risk of mortality among GCA cases (highest $v$ s lowest quartile $\mathrm{HR}=0.63,95 \% \mathrm{CI}=0.42-0.95, P_{\text {trend }}=0.25$ ) and more modestly among OEA cases ( $\mathrm{HR}=0.87,95 \% \mathrm{CI}=0.60-1.26)$, which included the null value in the CI. Dietary lignan intake was associated with decreased mortality for OES cases ( $\mathrm{HR}=0.58,95 \% \mathrm{CI}=0.37$ $\left.0.92, P_{\text {trend }}=0.07\right)$ and more modestly for OEA cases $(\mathrm{HR}=0.78$, $95 \% \mathrm{CI}=0.54-1.14)$, with the $\mathrm{CI}$ including the null value. Flavanone intake was associated with decreased mortality for OGA cases $\left(\mathrm{HR}=0.66,95 \% \mathrm{CI}=0.46-0.93, P_{\text {trend }}=0.18\right)$. Flavone intake was associated with modest decreased mortality for OEA cases $(\mathrm{HR}=0.83,95 \% \mathrm{CI}=0.58-1.19)$, but the $\mathrm{CI}$ included the null. In addition, total flavonoid or flavonol intake was associated with little or no decreased risk of mortality for any tumour type.

A sensitivity analysis that excluded the anthocyanidin value for bananas did not substantially alter our results for oesophageal or gastric cancer incidence or survival (Supplementary Tables 6-9).

DISCUSSION

This is the first population-based study to examine associations between dietary flavonoid intake and survival among oesophageal and gastric cancer cases. In our analysis, the highest intake quartile of anthocyanidins was associated with a possible $37 \%$ and more modest $13 \%$ decreased risk of mortality for GCA and OEA cases, respectively. Lignan intake was associated with a possible $42 \%$ and more modest $22 \%$ decreased risk of mortality for OES and OEA 
Table 2. Major sources of flavonoids and lignans among a population-based sample of control participants without oesophageal or gastric cancer with information on dietary intake, USA Multicenter Study: Connecticut, New Jersey, and Western Washington State, 1993-1995

\begin{tabular}{|c|c|c|}
\hline $\begin{array}{l}\text { Flavonoid/ } \\
\text { phytoestrogen class }\end{array}$ & Representative flavonoids & Main FFQ line item sources (\%) \\
\hline Total flavonoids & & Black tea (55.8), orange/grapefruit juice (14.2), wine (4.5) \\
\hline Anthocyanidins & $\begin{array}{l}\text { Cyanidin, Delphinidin, Malvidin, } \\
\text { Pelargonidin, Peonidin, Petunidin }\end{array}$ & $\begin{array}{l}\text { Wine (29.1), banana (13.7), fruit juice (10.6), other fruit (fruit cocktail, grapes, pineapple, } \\
\text { blueberries, applesauce) (9.0), apples (7.5), pies (6.0), bean soup (5.9) }\end{array}$ \\
\hline Flavan-3-ols & $\begin{array}{l}\text { (+)-Catechin, (+)-Catechin-3-gallate, } \\
\text { (-)-Epicatechin, (-)-Epicatechin-3- } \\
\text { gallate, (-)-Epigallocatechin, } \\
(-) \text {-Epigallocatechin- 3-gallate, } \\
(+) \text {-Gallocatechin, (+)-Gallocatechin- } \\
\text { 3-gallate, Theaflavin, Theaflavin-3- } \\
\text { gallate, Theaflavin-3'-gallate, } \\
\text { Theaflavin-3,3'-digallate, } \\
\text { Thearubigins }\end{array}$ & $\begin{array}{l}\text { Black tea (83.7), beer (3.2), green tea (3.1), wine (2.0), apples (1.9), } \\
\text { bananas (1.4) }\end{array}$ \\
\hline Flavanones & Eriodictyol, Hesperetin, Naringenin & Orange/grapefruit juice (75.4), oranges (21.5), wine (1.8) \\
\hline Flavones & Apigenin, Luteolin & $\begin{array}{l}\text { Pizza ( } 27.5) \text {, Wine (13.0), Celery (8.3), vegetable soup (7.8), mixed salad (7.8), cream soups } \\
\text { (7.0), chicken noodle soup (5.4) }\end{array}$ \\
\hline Flavonols & $\begin{array}{l}\text { Isorhamnetin, Kaempferol, Myricetin, } \\
\text { Quercetin }\end{array}$ & Black tea (22.9), onions (10.5), beer (9.2), apples (8.1), wine (5.9), mixed salad (5.9) \\
\hline Isoflavones & Daidzein, Genistein, Glycitein & Coffee (36.8), chili (17.4), white bread (10.8), cake (8.4), fried chicken (5.1), sausage (4.5) \\
\hline Lignans & Matairesinol, Secoisolariciresinol & Coffee (34.8), orange/grapefruit juice (13.4), wine (10.3), black tea (5.0), onions (3.5) \\
\hline
\end{tabular}

cases, respectively. Flavanones was associated with a possible $34 \%$ decreased risk of mortality for OGA cases. We also found flavones associated with a possible $17 \%$ decreased risk of mortality for OEA cases. Although our survival results are intriguing, there are several aspects regarding our study approach (as discussed below) that should be kept in mind when interpreting our study findings.

In addition, we observed possible $25-30 \%$ risk reduction for the associations between anthocyanidins and GCA and OGA incidence and lignans and OGA incidence. We observed $\sim 30-60 \%$ risk reductions for the associations between anthocyanidins and flavanones and OEA incidence; similar risk reductions were noted for the associations between the highest intake quartiles of anthocyanidins, flavanones, flavones, isoflavones, and lignans in relation to OES. As these four cancer types have differing risk factors (Hongo et al, 2009; de Martel et al, 2013), it is not surprising that certain flavonoids differentially influence the risk of certain subtypes of oesophageal or gastric cancer.

Our findings are consistent with a previous epidemiologic study that reported an inverse association between anthocyanidin intake and OEA incidence (Bobe et al, 2009). In addition, our findings regarding anthocyanidin intake are consistent with the parent (Gammon et al, 1997) and consortial studies (Freedman et al, 2011) showing a risk reduction for moderate wine intake, the main source of anthocyanidins in this study. Our results are also consistent with previous population studies regarding flavanone and isoflavone intake in relation to OES incidence (Rossi et al, 2007; Bobe et al, 2009). Previous studies, but not our study reported here, have also noted inverse associations between: flavonols and OES incidence (Rossi et al, 2007); flavonols (Lagiou et al, 2004; Rossi et al, 2010), flavanones (Lagiou et al, 2004), flavones (Lagiou et al, 2004), and lignans (secoisolariciresinol; Hernandez-Ramirez et al, 2009) and gastric adenocarcinoma (i.e., cardia and noncardia) incidence; and flavonols (quercetin) and GCA incidence (Ekstrom et al, 2011). Observed differences between these previous studies and our own could partially be because of considering gastric adenocarcinoma as a combined entity and not according to anatomic subsite, examining single flavonoids and not classes of flavonoids, differences in study populations and dietary patterns across studies, or chance.

In this study, notable risk reductions were observed for the associations between anthocyanidins and oesophageal cancer incidence, regardless of histologic subtype. However, in animal studies, results for anthocyanidins as chemopreventive agents are not consistent across histologic subtypes (i.e., squamous cell carcinoma $v s$ adenocarcinoma). Multiple experimental studies have shown that anthocyanidin-rich black raspberries have chemopreventive properties in $N$-nitrosomethylbenzylamine-induced tumours in rats (OES rodent model) (Stoner et al, 2006). In a study of oesophagoduodenal anastomosis, a rodent model for reflux-induced OEA, freeze-dried black raspberries were not effective in chemoprevention of OEA (Aiyer et al, 2011). However, interim clinical trial results for Barrett's oesophagus, a potential precursor of OEA, found reduced markers of oxidative stress in Barrett's oesophagus patients consuming anthocyanidin-rich freeze-dried black raspberries (Kresty et al, 2006).

A previous study, based on data from the same parent study as our own, found a modest inverse association between fruit and vegetable intake and incidence of both histologic subtypes of oesophageal cancer (Navarro Silvera et al, 2008). As flavonoids are concentrated in fruits and vegetables (D'Archivio et al, 2007), the association between flavonoids and oesophageal or gastric cancer incidence may reflect diets with greater consumption of such foods or a healthy lifestyle in general. The parent study assessed many lifestyle factors, including cigarette smoking, alcohol intake, and BMI, individually (Gammon et al, 1997) and through the use of pattern analyses (Navarro Silvera et al, 2008). Cigarette smoking was the only covariate among these that influenced our findings and was adjusted for in the final models. Thus, lifestyle seems unlikely to account for our results.

When estimating flavonoid content in food, particularly fruits and vegetables, potential sources of error include plant varieties, degree of ripeness, storage conditions, distance transported to market, environmental factors affecting plant growth, horticultural practices, industrial processing, and cooking methods that may vary over time and by geographic region (D'Archivio et al, 2007). Therefore, the foods utilised to create the nutrient database 
Table 3. Adjusted ${ }^{\mathrm{a}}$ odds ratios (ORs) and $95 \%$ confidence intervals (Cls) for associations between flavonoid and lignan intakes and oesophageal and gastric cancer incidence by tumour type, USA Multicenter Study: Connecticut, New Jersey, and Western Washington State, 1993-1995

\begin{tabular}{|c|c|c|c|c|c|c|c|c|c|}
\hline \multirow[b]{2}{*}{$\begin{array}{l}\text { Variable } \\
\text { and intake } \\
\text { (mg per day) }\end{array}$} & \multirow[b]{2}{*}{$\begin{array}{c}\text { Controls } \\
(N=662)\end{array}$} & \multicolumn{2}{|c|}{$\begin{array}{c}\text { Oesophageal } \\
\text { adenocarcinoma (OEA) }\end{array}$} & \multicolumn{2}{|c|}{$\begin{array}{c}\text { Gastric cardia } \\
\text { adenocarcinoma (GCA) }\end{array}$} & \multicolumn{2}{|c|}{$\begin{array}{l}\text { Oesophageal squamous } \\
\text { cell carcinoma (OES) }\end{array}$} & \multicolumn{2}{|c|}{$\begin{array}{c}\text { Other gastric } \\
\text { adenocarcinoma (OGA) }\end{array}$} \\
\hline & & $\begin{array}{c}\text { Cases } \\
(N=274)\end{array}$ & $\begin{array}{c}\text { OR } \\
(95 \% \mathrm{Cl})\end{array}$ & $\begin{array}{c}\text { Cases } \\
(N=248)\end{array}$ & $\begin{array}{c}\text { OR } \\
(95 \% \mathrm{Cl})\end{array}$ & $\begin{array}{c}\text { Cases } \\
(N=191)\end{array}$ & $\begin{array}{c}\text { OR } \\
(95 \% \mathrm{Cl})\end{array}$ & $\begin{array}{c}\text { Cases } \\
(N=341)\end{array}$ & $\begin{array}{c}\text { OR } \\
(95 \% \mathrm{Cl})\end{array}$ \\
\hline \multicolumn{10}{|c|}{ Total flavonoids } \\
\hline $\begin{array}{l}0-63.81 \\
63.82-97.90 \\
97.91-217.35 \\
\geqslant 217.36 \\
P \text { for trend }^{d}\end{array}$ & $\begin{array}{l}165 \\
166 \\
165 \\
166\end{array}$ & $\begin{array}{l}83 \\
54 \\
57 \\
80\end{array}$ & $\begin{array}{c}1.00 \\
0.62(0.41,0.93)^{b, c} \\
0.64(0.42,0.97) \\
0.92(0.63,1.37) \\
0.61\end{array}$ & $\begin{array}{l}59 \\
60 \\
54 \\
75\end{array}$ & $\begin{array}{c}1.00 \\
1.00(0.65,1.54) \\
0.90(0.58,1.41) \\
1.32(0.87,2.00) \\
0.07\end{array}$ & $\begin{array}{l}48 \\
35 \\
61 \\
47\end{array}$ & $\begin{array}{c}1.00 \\
0.64(0.38,1.07)^{\mathbf{b}} \\
1.11(0.69,1.77) \\
0.87(0.53,1.41) \\
0.42\end{array}$ & $\begin{array}{l}80 \\
84 \\
78 \\
99\end{array}$ & $\begin{array}{c}1.00 \\
1.01(0.69,1.49)^{c} \\
0.92(0.62,1.37) \\
1.08(0.73,1.58) \\
0.50\end{array}$ \\
\hline \multicolumn{10}{|c|}{ Anthocyanidins } \\
\hline $\begin{array}{l}0-7.21 \\
7.22-11.53 \\
11.54-18.47 \\
\geqslant 18.48 \\
P \text { for trend }^{d}\end{array}$ & $\begin{array}{l}166 \\
165 \\
165 \\
166\end{array}$ & $\begin{array}{l}98 \\
69 \\
59 \\
48\end{array}$ & $\begin{array}{c}1.00 \\
0.65(0.45,0.96) \\
0.54(0.36,0.81)^{c, e} \\
0.43(0.29,0.66) \\
\quad 0.06^{c, e}\end{array}$ & $\begin{array}{l}67 \\
67 \\
63 \\
51\end{array}$ & $\begin{array}{c}1.00 \\
0.98(0.65,1.47) \\
0.91(0.60,1.38)^{\mathrm{e}} \\
0.71(0.46,1.10) \\
0.91^{\mathrm{e}}\end{array}$ & $\begin{array}{l}67 \\
51 \\
37 \\
36\end{array}$ & $\begin{array}{c}1.00 \\
0.68(0.43,1.06) \\
0.44(0.27,0.72) \\
0.43(0.26,0.70) \\
0.11\end{array}$ & $\begin{array}{l}92 \\
89 \\
91 \\
69\end{array}$ & $\begin{array}{c}1.00 \\
0.91(0.63,1.32) \\
0.89(0.61,1.29)^{c} \\
0.70(0.47,1.03) \\
0.10^{c}\end{array}$ \\
\hline \multicolumn{10}{|l|}{ Flavan-3-ols } \\
\hline $\begin{array}{l}0-10.29 \\
10.30-22.00 \\
22.01-130.69 \\
\geqslant 130.70 \\
P \text { for trend }^{d}\end{array}$ & $\begin{array}{l}165 \\
166 \\
165 \\
166\end{array}$ & $\begin{array}{l}75 \\
61 \\
56 \\
82\end{array}$ & $\begin{array}{c}1.00 \\
0.72(0.48,1.09)^{c} \\
0.65(0.43,1.00) \\
1.02(0.69,1.51) \\
0.32\end{array}$ & $\begin{array}{l}59 \\
50 \\
69 \\
70\end{array}$ & $\begin{array}{c}1.00 \\
0.77(0.50,1.20) \\
1.05(0.69,1.61) \\
1.17(0.77,1.78) \\
0.07\end{array}$ & $\begin{array}{l}45 \\
37 \\
62 \\
47\end{array}$ & $\begin{array}{c}1.00 \\
0.78(0.47,1.30) \\
1.24(0.77,2.00) \\
0.98(0.60,1.59) \\
0.68\end{array}$ & $\begin{array}{c}71 \\
101 \\
68 \\
101\end{array}$ & $\begin{array}{c}1.00 \\
1.45(0.99,2.14)^{c} \\
0.98(0.65,1.48) \\
1.30(0.88,1.92) \\
0.36\end{array}$ \\
\hline \multicolumn{10}{|l|}{ Flavanones } \\
\hline $\begin{array}{l}0-11.57 \\
11.58-34.95 \\
34.96-49.52 \\
\geqslant 49.53 \\
P \text { for trend }^{d}\end{array}$ & $\begin{array}{l}166 \\
165 \\
165 \\
166\end{array}$ & $\begin{array}{l}91 \\
61 \\
69 \\
53\end{array}$ & $\begin{array}{c}1.00 \\
0.67(0.45,1.00) \\
0.75(0.50,1.10) \\
0.56(0.37,0.85)^{\mathrm{e}} \\
0.003^{\mathrm{e}}\end{array}$ & $\begin{array}{l}62 \\
54 \\
59 \\
73\end{array}$ & $\begin{array}{c}1.00 \\
0.86(0.56,1.33) \\
1.01(0.66,1.55) \\
1.23(0.81,1.87)^{\mathrm{e}} \\
0.82^{\mathrm{e}}\end{array}$ & $\begin{array}{l}61 \\
45 \\
49 \\
36\end{array}$ & $\begin{array}{c}1.00 \\
0.70(0.44,1.11) \\
0.69(0.43,1.10) \\
0.48(0.29,0.78) \\
0.002\end{array}$ & $\begin{array}{l}89 \\
80 \\
83 \\
89\end{array}$ & $\begin{array}{c}1.00 \\
0.92(0.63,1.35) \\
0.86(0.59,1.27) \\
0.88(0.60,1.28) \\
0.36\end{array}$ \\
\hline \multicolumn{10}{|l|}{ Flavones } \\
\hline $\begin{array}{l}0-1.29 \\
1.30-1.90 \\
1.91-2.62 \\
\geqslant 2.63 \\
P \text { for trend }^{d}\end{array}$ & $\begin{array}{l}165 \\
166 \\
166 \\
165\end{array}$ & $\begin{array}{l}82 \\
58 \\
59 \\
75\end{array}$ & $\begin{array}{c}1.00 \\
0.67(0.45,1.00) \\
0.68(0.45,1.02)^{b} \\
0.84(0.56,1.25) \\
0.81^{b}\end{array}$ & $\begin{array}{l}60 \\
55 \\
62 \\
71\end{array}$ & $\begin{array}{c}1.00 \\
0.87(0.57,1.34) \\
0.98(0.64,1.50) \\
1.09(0.71,1.67) \\
0.15\end{array}$ & $\begin{array}{l}71 \\
51 \\
28 \\
41\end{array}$ & $\begin{array}{c}1.00 \\
0.70(0.45,1.09) \\
0.34(0.20,0.57)^{b} \\
0.55(0.34,0.89) \\
0.02^{b}\end{array}$ & $\begin{array}{l}95 \\
87 \\
72 \\
87\end{array}$ & $\begin{array}{c}1.00 \\
0.92(0.64,1.34) \\
0.77(0.52,1.13) \\
1.01(0.69,1.50) \\
0.98\end{array}$ \\
\hline \multicolumn{10}{|l|}{ Flavonols } \\
\hline $\begin{array}{l}0-8.31 \\
8.32-12.16 \\
12.17-17.81 \\
\geqslant 17.82 \\
P \text { for trend }^{d}\end{array}$ & $\begin{array}{l}166 \\
165 \\
166 \\
165\end{array}$ & $\begin{array}{l}86 \\
49 \\
61 \\
78\end{array}$ & $\begin{array}{c}1.00 \\
0.56(0.37,0.85)^{\mathrm{e}, \mathrm{f}} \\
0.67(0.45,1.00) \\
0.80(0.54,1.18)^{\mathbf{e}} \\
0.71\end{array}$ & $\begin{array}{l}52 \\
64 \\
53 \\
79\end{array}$ & $\begin{array}{c}1.00 \\
1.24(0.81,1.91)^{\mathrm{e}} \\
1.01(0.65,1.57) \\
1.42(0.93,2.17)^{\mathrm{e}} \\
0.10\end{array}$ & $\begin{array}{l}56 \\
33 \\
39 \\
63\end{array}$ & $\begin{array}{c}1.00 \\
0.59(0.36,0.98) \\
0.70(0.43,1.14) \\
0.97(0.62,1.53) \\
0.70\end{array}$ & $\begin{array}{l}82 \\
94 \\
79 \\
86\end{array}$ & $\begin{array}{c}1.00 \\
1.16(0.80,1.69)^{c} \\
0.96(0.65,1.41) \\
0.98(0.67,1.46) \\
0.92\end{array}$ \\
\hline \multicolumn{10}{|l|}{ Isoflavones } \\
\hline $\begin{array}{l}0-0.27 \\
0.28-0.41 \\
0.42-0.59 \\
\geqslant 0.60 \\
P \text { for trend }\end{array}$ & $\begin{array}{l}165 \\
166 \\
166 \\
165\end{array}$ & $\begin{array}{l}47 \\
51 \\
83 \\
93\end{array}$ & $\begin{array}{c}1.00 \\
0.98(0.61,1.55) \\
1.51(0.97,2.37) \\
1.65(1.02,2.65)^{b} \\
0.07^{b}\end{array}$ & $\begin{array}{l}36 \\
64 \\
73 \\
75\end{array}$ & $\begin{array}{c}1.00 \\
1.55(0.97,2.49) \\
1.62(1.00,2.63) \\
1.56(0.93,2.60) \\
0.17\end{array}$ & $\begin{array}{l}34 \\
50 \\
59 \\
48\end{array}$ & $\begin{array}{c}1.00 \\
1.12(0.67,1.89) \\
1.18(0.69,2.00) \\
0.72(0.40,1.29)^{\mathbf{b}} \\
0.11^{b}\end{array}$ & $\begin{array}{l}65 \\
90 \\
97 \\
89\end{array}$ & $\begin{array}{c}1.00 \\
1.45(0.97,2.17) \\
1.68(1.10,2.56) \\
1.50(0.96,2.37) \\
0.37\end{array}$ \\
\hline \multicolumn{10}{|l|}{ Lignans } \\
\hline $\begin{array}{l}0-0.045 \\
0.046-0.063 \\
0.064-0.082 \\
\geqslant 0.083 \\
P \text { for trend }^{d}\end{array}$ & $\begin{array}{l}165 \\
166 \\
165 \\
166\end{array}$ & $\begin{array}{l}70 \\
75 \\
57 \\
72\end{array}$ & $\begin{array}{c}1.00 \\
0.91(0.61,1.36) \\
0.65(0.42,0.99)^{c, e} \\
0.75(0.49,1.13)^{b} \\
0.26^{b}\end{array}$ & $\begin{array}{l}50 \\
61 \\
70 \\
67\end{array}$ & $\begin{array}{c}1.00 \\
1.06(0.69,1.65) \\
1.17(0.76,1.81)^{\mathrm{e}} \\
1.01(0.65,1.58) \\
0.63\end{array}$ & $\begin{array}{l}67 \\
47 \\
42 \\
35\end{array}$ & $\begin{array}{c}1.00 \\
0.64(0.40,1.01) \\
0.50(0.31,0.82) \\
0.38(0.23,0.63)^{b} \\
0.0003^{b}\end{array}$ & $\begin{array}{l}87 \\
92 \\
98 \\
64\end{array}$ & $\begin{array}{c}1.00 \\
1.09(0.75,1.59) \\
1.11(0.76,1.63)^{\mathrm{c}} \\
0.73(0.48,1.11) \\
0.13\end{array}$ \\
\hline \multicolumn{10}{|c|}{ 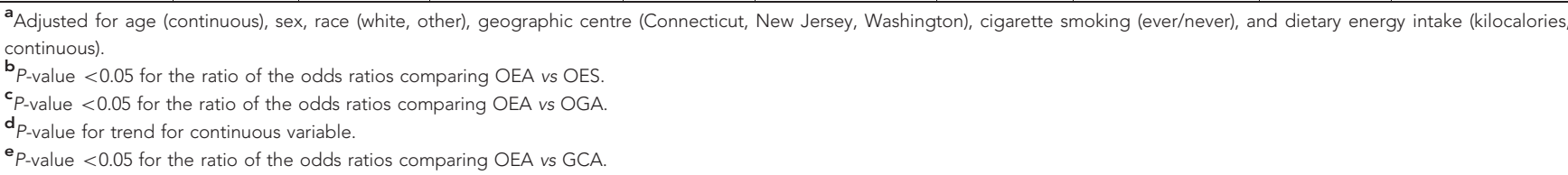 } \\
\hline
\end{tabular}

estimates may differ from the actual food sources reportedly consumed by this study population (Thompson et al, 2006; Bhagwat et al, 2008, 2011). To estimate the potential impact of such influences, the USDA Food Composition and Nutrient Data Laboratories sampled over 60 fruits, vegetables, and nuts from four USA regions at two times of the year and estimated the flavonoid content. Values reported in the USDA databases were similar to average flavonoid content determined in this study, although high variability of flavonoid content was seen within and between foods (Harnly et al, 2006). In addition, the FFQ line item for wine did not distinguish between red and white that have different flavonoid concentrations (Bhagwat et al, 2011). In our study reported here, weights of $50 \%$ for red and $50 \%$ for white were assigned. However, individuals often preferentially drink red or white wine, and thus individual estimates of flavonoid types for which wine is a source may be misclassified.

Bioavailability of flavonoid compounds is another potential source of error when estimating the amount of flavonoid intake necessary to reduce risk of oesophageal or gastric cancer. However, little is known about flavonoid absorption in the gastrointestinal tract, metabolism of flavonoids varies by individual, and the degree to which flavonoids might have direct effects on epithelial surfaces 
Table 4. Adjusted ${ }^{\mathrm{a}}$ hazard ratios (HRs) and $95 \%$ confidence intervals (Cls) for flavonoid and lignan intakes and overall mortality in oesophageal or gastric cancer cases by tumour type, USA Multicenter Study: Connecticut, New Jersey, and Western Washington State, 1993-1995

\begin{tabular}{|c|c|c|c|c|c|c|c|c|}
\hline \multirow[b]{2}{*}{$\begin{array}{l}\text { Variable and } \\
\text { intake } \\
\text { (mg per day) }\end{array}$} & \multicolumn{2}{|c|}{$\begin{array}{c}\text { Oesophageal } \\
\text { adenocarcinoma (OEA) }\end{array}$} & \multicolumn{2}{|c|}{$\begin{array}{c}\text { Gastric cardia } \\
\text { adenocarcinoma (GCA) }\end{array}$} & \multicolumn{2}{|c|}{$\begin{array}{l}\text { Oesophageal squamous } \\
\text { cell carcinoma (OES) }\end{array}$} & \multicolumn{2}{|c|}{$\begin{array}{c}\text { Other gastric } \\
\text { adenocarcinoma (OGA) }\end{array}$} \\
\hline & $\begin{array}{c}\text { Cases } \\
(N=274)\end{array}$ & $\begin{array}{c}\mathrm{HR} \\
(95 \% \mathrm{Cl})\end{array}$ & $\begin{array}{c}\text { Cases } \\
(N=248)\end{array}$ & $\begin{array}{c}\mathrm{HR} \\
(95 \% \mathrm{Cl})\end{array}$ & $\begin{array}{c}\text { Cases } \\
(N=191)\end{array}$ & $\begin{array}{c}\mathrm{HR} \\
(95 \% \mathrm{Cl})\end{array}$ & $\begin{array}{c}\text { Cases } \\
(N=338)\end{array}$ & $\begin{array}{c}\mathrm{HR} \\
(95 \% \mathrm{Cl})\end{array}$ \\
\hline \multicolumn{9}{|c|}{ Total flavonoids } \\
\hline $\begin{array}{l}0-62.35 \\
62.36-103.39 \\
103.40-253.24 \\
\geqslant 253.25 \\
P \text { for trend }\end{array}$ & $\begin{array}{l}83 \\
60 \\
65 \\
66\end{array}$ & $\begin{array}{c}1.00 \\
1.37(0.95,1.98) \\
1.39(0.97,1.98)^{b, c} \\
0.98(0.68,1.41) \\
0.11\end{array}$ & $\begin{array}{l}57 \\
66 \\
60 \\
65\end{array}$ & $\begin{array}{c}1.00 \\
1.05(0.71,1.54) \\
0.79(0.54,1.17)^{b} \\
0.88(0.59,1.32) \\
0.80\end{array}$ & $\begin{array}{l}47 \\
42 \\
61 \\
41\end{array}$ & $\begin{array}{c}1.00 \\
1.26(0.81,1.97) \\
0.92(0.60,1.40) \\
0.91(0.58,1.44) \\
0.11\end{array}$ & $\begin{array}{l}76 \\
95 \\
77 \\
90\end{array}$ & $\begin{array}{c}1.00 \\
1.01(0.71,1.42) \\
0.72(0.50,1.05)^{c} \\
0.99(0.70,1.39) \\
0.90\end{array}$ \\
\hline \multicolumn{9}{|c|}{ Anthocyanidins } \\
\hline $\begin{array}{l}0-6.23 \\
6.24-10.11 \\
10.12-16.23 \\
\geqslant 16.24 \\
P \text { for trend }\end{array}$ & $\begin{array}{l}83 \\
61 \\
67 \\
63\end{array}$ & $\begin{array}{c}1.00 \\
0.93(0.65,1.35) \\
0.88(0.62,1.26) \\
0.87(0.60,1.26) \\
0.14\end{array}$ & $\begin{array}{l}52 \\
64 \\
66 \\
66\end{array}$ & $\begin{array}{c}1.00 \\
0.76(0.51,1.12) \\
0.80(0.54,1.18) \\
0.63(0.42,0.95) \\
0.25\end{array}$ & $\begin{array}{l}57 \\
52 \\
41 \\
41\end{array}$ & $\begin{array}{c}1.00 \\
0.77(0.50,1.18) \\
1.46(0.94,2.26) \\
1.01(0.66,1.56) \\
0.94\end{array}$ & $\begin{array}{l}70 \\
86 \\
89 \\
93\end{array}$ & $\begin{array}{c}1.00 \\
1.27(0.89,1.82) \\
1.22(0.84,1.76) \\
1.14(0.80,1.63) \\
0.55\end{array}$ \\
\hline \multicolumn{9}{|l|}{ Flavan-3-ols } \\
\hline $\begin{array}{l}0-10.90 \\
10.91-26.67 \\
26.68-210.51 \\
\geqslant 210.52 \\
P \text { for trend }\end{array}$ & $\begin{array}{l}80 \\
62 \\
66 \\
66\end{array}$ & $\begin{array}{c}1.00 \\
0.92(0.64,1.32) \\
1.20(0.84,1.72)^{\mathrm{e}} \\
0.93(0.65,1.33) \\
0.11\end{array}$ & $\begin{array}{l}61 \\
53 \\
71 \\
63\end{array}$ & $\begin{array}{c}1.00 \\
0.69(0.46,1.04) \\
0.78(0.54,1.13) \\
0.71(0.48,1.05) \\
0.94\end{array}$ & $\begin{array}{l}47 \\
46 \\
56 \\
42\end{array}$ & $\begin{array}{c}1.00 \\
1.68(1.08,2.62) \\
0.97(0.63,1.50)^{\mathbf{e}} \\
1.09(0.69,1.74) \\
0.10\end{array}$ & $\begin{array}{r}75 \\
102 \\
70 \\
91\end{array}$ & $\begin{array}{c}1.00 \\
1.33(0.94,1.87) \\
0.96(0.66,1.39) \\
1.22(0.87,1.73) \\
0.80\end{array}$ \\
\hline \multicolumn{9}{|l|}{ Flavanones } \\
\hline $\begin{array}{l}0-8.63 \\
8.64-32.94 \\
34.95-49.00 \\
\geqslant 49.01 \\
P \text { for trend }\end{array}$ & $\begin{array}{l}81 \\
66 \\
71 \\
56\end{array}$ & $\begin{array}{c}1.00 \\
0.96(0.67,1.38) \\
1.40(0.99,1.98)^{c} \\
1.15(0.79,1.68)^{c} \\
0.05^{c}\end{array}$ & $\begin{array}{l}55 \\
60 \\
57 \\
76\end{array}$ & $\begin{array}{c}1.00 \\
1.12(0.75,1.69) \\
1.24(0.83,1.86) \\
0.90(0.61,1.33) \\
0.50\end{array}$ & $\begin{array}{l}50 \\
54 \\
48 \\
39\end{array}$ & $\begin{array}{c}1.00 \\
0.95(0.61,1.47) \\
1.05(0.67,1.63) \\
1.24(0.76,2.03) \\
0.80\end{array}$ & $\begin{array}{l}77 \\
83 \\
87 \\
91\end{array}$ & $\begin{array}{c}1.00 \\
0.70(0.49,1.00) \\
0.74(0.52,1.05)^{c} \\
0.66(0.46,0.93)^{c} \\
0.18^{c}\end{array}$ \\
\hline \multicolumn{9}{|l|}{ Flavones } \\
\hline $\begin{array}{l}0-1.20 \\
1.21-1.81 \\
1.82-2.64 \\
\geqslant 2.65 \\
P \text { for trend }\end{array}$ & $\begin{array}{l}79 \\
52 \\
73 \\
70\end{array}$ & $\begin{array}{c}1.00 \\
0.97(0.66,1.41) \\
0.89(0.63,1.26) \\
0.83(0.58,1.19) \\
0.04\end{array}$ & $\begin{array}{l}46 \\
63 \\
70 \\
69\end{array}$ & $\begin{array}{c}1.00 \\
1.03(0.68,1.56) \\
0.95(0.63,1.44) \\
0.86(0.56,1.31) \\
0.54\end{array}$ & $\begin{array}{l}64 \\
48 \\
39 \\
40\end{array}$ & $\begin{array}{c}1.00 \\
1.23(0.82,1.84) \\
0.88(0.56,1.38) \\
1.00(0.64,1.54) \\
0.17\end{array}$ & $\begin{array}{r}74 \\
100 \\
80 \\
84\end{array}$ & $\begin{array}{c}1.00 \\
0.88(0.63,1.24) \\
0.88(0.61,1.26) \\
0.97(0.67,1.39) \\
0.62\end{array}$ \\
\hline \multicolumn{9}{|l|}{ Flavonols } \\
\hline $\begin{array}{l}0-8.16 \\
8.17-12.30 \\
12.31-19.34 \\
\geqslant 19.35 \\
P \text { for trend }\end{array}$ & $\begin{array}{l}79 \\
60 \\
72 \\
63\end{array}$ & $\begin{array}{c}1.00 \\
1.30(0.89,1.89) \\
1.01(0.71,1.43) \\
0.94(0.65,1.37) \\
0.05\end{array}$ & $\begin{array}{l}50 \\
68 \\
63 \\
67\end{array}$ & $\begin{array}{c}1.00 \\
1.25(0.83,1.87) \\
1.11(0.74,1.68) \\
0.93(0.61,1.40) \\
0.35\end{array}$ & $\begin{array}{l}55 \\
36 \\
45 \\
55\end{array}$ & $\begin{array}{c}1.00 \\
1.14(0.70,1.83) \\
0.78(0.50,1.20) \\
0.93(0.61,1.40) \\
0.69\end{array}$ & $\begin{array}{l}79 \\
98 \\
84 \\
77\end{array}$ & $\begin{array}{c}1.00 \\
1.12(0.80,1.57) \\
1.09(0.78,1.54) \\
0.97(0.68,1.38) \\
0.71\end{array}$ \\
\hline \multicolumn{9}{|l|}{ Isoflavones } \\
\hline $\begin{array}{l}0-0.31 \\
0.32-0.46 \\
0.47-0.62 \\
\geqslant 0.63 \\
P \text { for trend }^{d}\end{array}$ & $\begin{array}{l}65 \\
56 \\
73 \\
80\end{array}$ & $\begin{array}{c}1.00 \\
1.00(0.68,1.47) \\
0.70(0.48,1.03) \\
0.75(0.49,1.13) \\
0.65\end{array}$ & $\begin{array}{l}59 \\
57 \\
68 \\
64\end{array}$ & $\begin{array}{c}1.00 \\
1.26(0.84,1.87) \\
0.94(0.62,1.44) \\
1.01(0.65,1.57) \\
0.77\end{array}$ & $\begin{array}{l}48 \\
60 \\
42 \\
41\end{array}$ & $\begin{array}{c}1.00 \\
1.03(0.68,1.58) \\
1.14(0.71,1.81) \\
0.97(0.60,1.58) \\
0.60\end{array}$ & $\begin{array}{l}89 \\
90 \\
81 \\
78\end{array}$ & $\begin{array}{c}1.00 \\
1.29(0.92,1.82) \\
0.86(0.60,1.25) \\
0.92(0.62,1.37) \\
0.88\end{array}$ \\
\hline \multicolumn{9}{|l|}{ Lignans } \\
\hline $\begin{array}{l}0-0.044 \\
0.045-0.060 \\
0.061-0.079 \\
\geqslant 0.080 \\
P \text { for trend }\end{array}$ & $\begin{array}{l}68 \\
72 \\
57 \\
77\end{array}$ & $\begin{array}{c}1.00 \\
0.85(0.60,1.23) \\
0.98(0.67,1.45) \\
0.78(0.54,1.14) \\
0.28\end{array}$ & $\begin{array}{l}50 \\
55 \\
66 \\
77\end{array}$ & $\begin{array}{c}1.00 \\
1.45(0.95,2.22) \\
1.05(0.70,1.59) \\
0.97(0.65,1.46) \\
0.43\end{array}$ & $\begin{array}{l}62 \\
50 \\
41 \\
38\end{array}$ & $\begin{array}{c}1.00 \\
0.73(0.47,1.13) \\
0.61(0.39,0.96) \\
0.58(0.37,0.92) \\
0.07\end{array}$ & $\begin{array}{l}83 \\
85 \\
99 \\
71\end{array}$ & $\begin{array}{c}1.00 \\
1.22(0.86,1.73) \\
1.08(0.77,1.51) \\
1.05(0.72,1.53) \\
0.55\end{array}$ \\
\hline \multicolumn{9}{|c|}{ 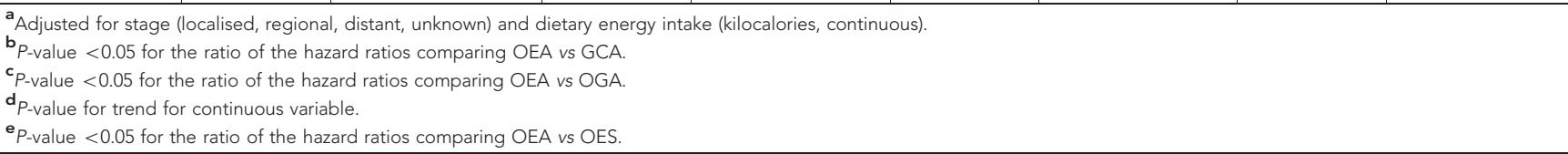 } \\
\hline
\end{tabular}

as they traverse the oesophagus and stomach is unclear (Bravo, 1998). In addition, absorption profiles of flavonoids vary, with maximum concentrations reached between 0.5 and $9 \mathrm{~h}$ after dietary intake (Spencer et al, 2008). Thus, serum flavonoid biomarkers may not be highly correlated with usual adult dietary intake. Although such variation in estimating representative dietary flavonoid intakes and bioavailability may be a study limitation, this issue would apply to greater or lesser degrees to all studies reliant on nutritional databases to estimate dietary intake (Willett, 2012).

Patients with gastroesophageal reflux disease or Barrett's oesophagus are recommended to omit foods that are mechanically or chemically irritating, including some flavonoid-rich foods 
(e.g., coffee, tea, alcohol, citrus, tomatoes, chocolate, peppers, and onions) (Ko et al, 2008). Although the FFQ assessed diet before diagnosis, OEA patients may already have symptoms before diagnosis, causing their usual diet to change or, perhaps, reporting of past diet could be influenced by current dietary habits. However, the foods that are irritating vary by individual (Brown, 2008); thus, we are unable to estimate how such potential changes in diet would have affected flavonoid intake values. In addition, a dietary study showed that intakes of fruits, vegetables, and alcohol did not differ by symptomatic gastroesophageal reflux disease status (El-Serag et al, 2005). Although it is unknown whether gastroesophageal reflux disease or Barrett's oesophagus are necessary precursors of OEA (Shaheen and Ransohoff, 2002), it is still possible that associations observed in case-control studies between flavonoid intake and risk of OEA are due to reverse causation. However, in examining the comparison case group of OES, we see similar associations, with the exception of isoflavones. Thus, it seems unlikely that our observed associations are completely because of reverse causation.

Although our observed association with oesophageal and gastric cancers could possibly reflect a true association with select flavonoid classes, or perhaps a healthy prediagnostic behaviour lifestyle in general, there are additional measurement issues to consider during the interpretation of our findings. For example, FFQ responses for the 3-5 years before diagnosis are assumed to reflect usual adult diet, both pre- and post-diagnosis. Whether such time period reflects intakes during the time relevant to oesophageal and gastric cancer development is unknown. However, because all existing oesophageal and gastric cancer studies have relied on FFQs (Lunet et al, 2007; Li et al, 2014), one would need to conduct a cohort study and employ multiple alternative dietary assessment methods over time to overcome limitations of existing studies. Such an alternative study design would be very inefficient, because the lifetime risk of oesophageal or gastric cancer in the general United States population is $<1 \%$ (Howlader et al). Similarly for the survival analyses, potential changes in dietary intake after diagnosis may be a relevant exposure but we were not able to assess this from available data.

Our study FFQ did not assess dietary supplement use of flavonoids. However, it is unlikely that use of flavonoid-rich supplements was widespread during this study time period, as flavonoid supplements were not patented in the United States until after completion of participant interviews in our parent study (U.S. Patent Office). In addition, we were unable to assess post-diagnosis medications or first course of treatment that may confound the survival analyses. Finally, the majority of participants were Caucasian males with low socioeconomic status. The overwhelming majority of individuals who develop oesophageal and gastric cancer are Caucasian males (Falk, 2009). Thus, the results are still largely generalisable to those at highest risk of developing these deadly cancers.

Multiple comparisons need to be considered when discussing the study results, as there were 64 comparisons within the main analyses, given we considered 8 exposures and 8 outcomes. Thus, there is a possibility that some statistically significant results arose because of chance. Adjusting for multiple comparisons would reduce the likelihood of detecting a false positive association, but would reduce power for detecting a true association if one exists. Instead, we chose to focus on associations based on biologic plausibility and consistency with published results (Rothman, 1990; Willett, 2012); we also gave more credence to results that were consistent across the continuum of cancer development. For example, in this study, we noted inverse associations for both incidence and survival for OEA in relation to anthocyanidin intake.

In summary, our population-based findings suggest that dietary intake of some types of flavonoids, particularly anthocyanidins, may lower the risk of oesophageal and gastric cancer incidence and may potentially enhance survival. This is the first epidemiologic study to examine the association of flavonoids and lignans with survival among oesophageal and gastric cancer cases, and one of few studies to examine these compounds in associations with the risk of incident oesophageal and gastric cancer by tumour type. Our findings here on anthocyanidins are consistent for incidence and survival of OEA and GCA, suggesting that these compounds could perhaps potentially be used in an effort to reduce mortality because of these fatal cancers. However, further research is needed before definite conclusions can be made about the chemopreventive role of dietary flavonoids and lignans on oesophageal and gastric cancer incidence and survival.

\section{ACKNOWLEDGEMENTS}

This research was supported in part by grants from the National Institutes of Health (R03-CA159409, T32-CA009330, T32ES007018, U01-CA057983, U01-CA057949, U01-CA057923, and P30-ES10126). We thank Eric Meier, Lynn Onstad, and Patricia Christopherson from the FHCRC for FFQ expertise; Dr Linda Liao from the National Cancer Institute for providing the parent study data; and Shelley Niwa from Westat for data preparation.

\section{CONFLICT OF INTEREST}

PEA participated in this research during predoctoral work at UNCChapel Hill and currently consults for various pharmaceutical companies. No money was received from any pharmaceutical company. The remaining authors declare no conflict of interest.

\section{DISCLAIMER}

The findings and conclusions in this report are those of the authors and do not necessarily represent the official position of the Centers for Disease Control and Prevention. Certain data used in this study were obtained from the Connecticut Tumor Registry, located in the Connecticut Department of Public Health. The authors assume full responsibility for analyses and interpretation of these data.

\section{REFERENCES}

Aiyer HS, Li Y, Losso JN, Gao C, Schiffman SC, Slone SP, Martin RC (2011) Effect of freeze-dried berries on the development of reflux-induced esophageal adenocarcinoma. Nutr Cancer 63(8): 1256-1262.

Bhagwat S, Haytowitz DB, Holden JM (2008) U.S. Department of Agriculture, Agricultural Research Service. USDA Database for the Isoflavone Content of Selected Foods, Release 2.0.

Bhagwat S, Haytowitz DB, Holden JM (2011) U.S. Department of Agriculture, Agricultural Research Service. USDA Database for the Flavonoid Content of Selected Foods, Release 3.0.

Bobe G, Peterson JJ, Gridley G, Hyer M, Dwyer JT, Brown LM (2009) Flavonoid consumption and esophageal cancer among black and white men in the United States. Int J Cancer 125(5): 1147-1154.

Bravo L (1998) Polyphenols: chemistry, dietary sources, metabolism, and nutritional significance. Nutr Rev 56(11): 317-333.

Brown JE (2008) Nutrition Through the Life Cycle. 3rd edn. Thomson/ Wadsworth: Belmont, CA.

D’Archivio M, Filesi C, Di Benedetto R, Gargiulo R, Giovannini C, Masella R (2007) Polyphenols, dietary sources and bioavailability. Ann Ist Super Sanita 43(4): 348-361.

de Martel C, Forman D, Plummer M (2013) Gastric cancer: epidemiology and risk factors. Gastroenterol Clin North Am 42(2): 219-240.

Devesa SS, Blot WJ, Fraumeni JF Jr (1998) Changing patterns in the incidence of esophageal and gastric carcinoma in the United States. Cancer 83(10): 2049-2053. 
Drossard C, Frohling B, Dietrich H, Kersting M (2011) Anthocyanin analysis in banana fruit-a mistake. Am J Clin Nutr 93(4): 865-866, author reply 866-7.

Ekstrom AM, Serafini M, Nyren O, Wolk A, Bosetti C, Bellocco R (2011) Dietary quercetin intake and risk of gastric cancer: results from a population-based study in Sweden. Ann Oncol 22(2): 438-443.

El-Serag HB, Satia JA, Rabeneck L (2005) Dietary intake and the risk of gastro-oesophageal reflux disease: a cross sectional study in volunteers. Gut 54(1): 11-17.

Falk GW (2009) Risk factors for esophageal cancer development. Surg Oncol Clin N Am 18(3): 469-485.

Freedman ND, Murray LJ, Kamangar F, Abnet CC, Cook MB, Nyren O, Ye W, Wu AH, Bernstein L, Brown LM, Ward MH, Pandeya N, Green AC, Casson AG, Giffen C, Risch HA, Gammon MD, Chow WH, Vaughan TL, Corley DA, Whiteman DC (2011) Alcohol intake and risk of oesophageal adenocarcinoma: a pooled analysis from the BEACON Consortium. Gut 60(8): 1029-1037.

Gammon MD, Schoenberg JB, Ahsan H, Risch HA, Vaughan TL, Chow WH, Rotterdam H, West AB, Dubrow R, Stanford JL, Mayne ST, Farrow DC, Niwa S, Blot WJ, Fraumeni JF Jr (1997) Tobacco, alcohol, and socioeconomic status and adenocarcinomas of the esophagus and gastric cardia. J Natl Cancer Inst 89(17): 1277-1284.

Harnly JM, Doherty RF, Beecher GR, Holden JM, Haytowitz DB, Bhagwat S, Gebhardt S (2006) Flavonoid content of U.S. fruits, vegetables, and nuts. J Agric Food Chem 54(26): 9966-9977.

Hernandez-Ramirez RU, Galvan-Portillo MV, Ward MH, Agudo A, Gonzalez CA, Onate-Ocana LF, Herrera-Goepfert R, Palma-Coca O, Lopez-Carrillo L (2009) Dietary intake of polyphenols, nitrate and nitrite and gastric cancer risk in Mexico City. Int J Cancer 125(6): $1424-1430$.

Hongo M, Nagasaki Y, Shoji T (2009) Epidemiology of esophageal cancer: Orient to Occident. Effects of chronology, geography and ethnicity. J Gastroenterol Hepatol 24(5): 729-735.

Howlader N, Noone AM, Krapcho M, Garshell J, Neyman N, Altekruse SF, Kosary CL, Yu M, Ruhl J, Tatalovich Z, Cho H, Mariotto A, Lewis DR, Chen HS, Feuer EJ, Cronin KAe. SEER Cancer Statistics Review, 1975-2010, National Cancer Institute. Bethesda, MD. http://seer.cancer. gov/csr/1975_2010/, based on November 2012 SEER data submission, posted to the SEER web site, April 2013. Accessed 16 December 2013.

Huang WY, Cai YZ, Zhang Y (2010) Natural phenolic compounds from medicinal herbs and dietary plants: potential use for cancer prevention. Nutr Cancer 62(1): 1-20.

Ko AH, Dollinger M, Rosenbaum EH (2008) Everyone's Guide to Cancer Therapy: How Cancer Is Diagnosed, Treated, and Managed Day to Day. Rev. 5th edn. Andrews McMeel Pub: Kansas City.

Kresty LA, Frankel WL, Hammond CD, Baird ME, Mele JM, Stoner GD, Fromkes JJ (2006) Transitioning from preclinical to clinical chemopreventive assessments of lyophilized black raspberries: interim results show berries modulate markers of oxidative stress in Barrett's esophagus patients. Nutr Cancer 54(1): 148-156.

Kristal AR, Feng Z, Coates RJ, Oberman A, George V (1997) Associations of race/ethnicity, education, and dietary intervention with the validity and reliability of a food frequency questionnaire: the Women's Health Trial Feasibility Study in Minority Populations. Am J Epidemiol 146(10): 856-869.

Lagiou P, Samoli E, Lagiou A, Peterson J, Tzonou A, Dwyer J, Trichopoulos D (2004) Flavonoids, vitamin C and adenocarcinoma of the stomach. Cancer Causes Control 15(1): 67-72.

Li B, Jiang G, Zhang G, Xue Q, Zhang H, Wang C, Zhao T (2014) Intake of vegetables and fruit and risk of esophageal adenocarcinoma: a meta-analysis of observational studies. Eur J Nutr 53(7): 1511-1521.

Lunet N, Valbuena C, Vieira AL, Lopes C, David L, Carneiro F, Barros H (2007) Fruit and vegetable consumption and gastric cancer by location and histological type: case-control and meta-analysis. Eur J Cancer Prev 16(4): 312-327.

Mayne ST, Risch HA, Dubrow R, Chow WH, Gammon MD, Vaughan TL, Farrow DC, Schoenberg JB, Stanford JL, Ahsan H, West AB, Rotterdam H, Blot WJ, Fraumeni JF Jr (2001) Nutrient intake and risk of subtypes of esophageal and gastric cancer. Cancer Epidemiol Biomarkers Prev 10(10): 1055-1062.

Navarro Silvera SA, Mayne ST, Risch H, Gammon MD, Vaughan TL, Chow WH, Dubrow R, Schoenberg JB, Stanford JL, West AB, Rotterdam H, Blot WJ, Fraumeni JF Jr (2008) Food group intake and risk of subtypes of esophageal and gastric cancer. Int $J$ Cancer 123(4): 852-860.

Pierini R, Kroon PA, Guyot S, Ivory K, Johnson IT, Belshaw NJ (2008) Procyanidin effects on oesophageal adenocarcinoma cells strongly depend on flavan-3-ol degree of polymerization. Mol Nutr Food Res 52(12): 1399-1407.

Rossi M, Garavello W, Talamini R, La Vecchia C, Franceschi S, Lagiou P, Zambon P, Dal Maso L, Bosetti C, Negri E (2007) Flavonoids and risk of squamous cell esophageal cancer. Int J Cancer 120(7): 1560-1564.

Rossi M, Rosato V, Bosetti C, Lagiou P, Parpinel M, Bertuccio P, Negri E, La Vecchia C (2010) Flavonoids, proanthocyanidins, and the risk of stomach cancer. Cancer Causes Control 21(10): 1597-1604.

Rothman KJ (1990) No adjustments are needed for multiple comparisons. Epidemiology 1(1): 43-46.

Rothman KJ, Greenland S, Lash TL (2008) Modern Epidemiology. 3rd edn. Wolters Kluwer Health/Lippincott Williams \& Wilkins: Philadelphia.

Schwartz GK, Ilson D, Saltz L, O’Reilly E, Tong W, Maslak P, Werner J, Perkins P, Stoltz M, Kelsen D (2001) Phase II study of the cyclindependent kinase inhibitor flavopiridol administered to patients with advanced gastric carcinoma. J Clin Oncol 19(7): 1985-1992.

Shaheen N, Ransohoff DF (2002) Gastroesophageal reflux, barrett esophagus, and esophageal cancer: scientific review. JAMA 287(15): 1972-1981.

Simard EP, Ward EM, Siegel R, Jemal A (2012) Cancers with increasing incidence trends in the United States: 1999 through 2008. CA Cancer J Clin 62(2): 118-128.

Spencer JP, Abd El Mohsen MM, Minihane AM, Mathers JC (2008) Biomarkers of the intake of dietary polyphenols: strengths, limitations and application in nutrition research. Br J Nutr 99(1): 12-22.

Stoner GD, Chen T, Kresty LA, Aziz RM, Reinemann T, Nines R (2006) Protection against esophageal cancer in rodents with lyophilized berries: potential mechanisms. Nutr Cancer 54(1): 33-46.

Sun CL, Yuan JM, Lee MJ, Yang CS, Gao YT, Ross RK, Yu MC (2002) Urinary tea polyphenols in relation to gastric and esophageal cancers: a prospective study of men in Shanghai, China. Carcinogenesis 23(9): 1497-1503.

Thompson LU, Boucher BA, Liu Z, Cotterchio M, Kreiger N (2006) Phytoestrogen content of foods consumed in Canada, including isoflavones, lignans, and coumestan. Nutr Cancer 54(2): 184-201.

Trivers KF, De Roos AJ, Gammon MD, Vaughan TL, Risch HA, Olshan AF, Schoenberg JB, Mayne ST, Dubrow R, Stanford JL, Abrahamson P, Rotterdam H, West AB, Fraumeni JF, Chow WH (2005) Demographic and lifestyle predictors of survival in patients with esophageal or gastric cancers. Clin Gastroenterol Hepatol 3(3): 225-230.

U.S. Patent Office. Extract from Ginkgo biloba leaves, its method of preparation and pharmaceuticals containing the extract. http:// patft.uspto.gov/netacgi/nph-Parser?Sect1=PTO1\&Sect2=HITOFF\&d $=$ PALL $\& \mathrm{p}=1 \& \mathrm{u}=\% 2 \mathrm{Fnetahtml} \% 2 \mathrm{FPTO} \% 2 \mathrm{Fsrchnum} . \mathrm{htm} \& \mathrm{r}=1 \& \mathrm{f}=\mathrm{G} \& \mathrm{l}=$ 50\&s1=5,399,348.PN.\&OS=PN/5,399,348\&RS=PN/5,399,348, Accessed 9 July 2013.

Vermeulen E, Zamora-Ros R, Duell EJ, Lujan-Barroso L, Boeing H, Aleksandrova K, Bueno-de-Mesquita HB, Scalbert A, Romieu I, Fedirko V, Touillaud M, Fagherazzi G, Perquier F, Molina-Montes E, Chirlaque MD, Vicente Arguelles M, Amiano P, Barricarte A, Pala V, Mattiello A, Saieva C, Tumino R, Ricceri F, Trichopoulou A, Vasilopoulou E, Ziara G, Crowe FL, Khaw KT, Wareham NJ, Lukanova A, Grote VA, Tjonneland A, Halkjaer J, Bredsdorff L, Overvad K, Siersema PD, Peeters PH, May AM, Weiderpass E, Skeie G, Hjartaker A, Landberg R, Johansson I, Sonestedt E, Ericson U, Riboli E, Gonzalez CA (2013) Dietary flavonoid intake and esophageal cancer risk in the European prospective investigation into cancer and nutrition cohort. Am J Epidemiol 178(4): $570-581$.

Waksberg J (1978) Sampling methods for random digit dialing. J Am Stat Assoc 73: 40-46.

Wijnhoven BP, Siersema PD, Hop WC, van Dekken H, Tilanus HW (1999) Adenocarcinomas of the distal oesophagus and gastric cardia are one clinical entity. Rotterdam Oesophageal Tumour Study Group. Br J Surg 86(4): 529-535.

Willett W (2012) Nutritional Epidemiology. Vol. third. Oxford University Press: New York.

Willett WC, Howe GR, Kushi LH (1997) Adjustment for total energy intake in epidemiologic studies. Am J Clin Nutr 65(4 Suppl): 1220S-1228S discussion 1229S-1231S. 
Zamora-Ros R, Agudo A, Lujan-Barroso L, Romieu I, Ferrari P, Knaze V, Bueno-de-Mesquita HB, Leenders M, Travis RC, Navarro C, SanchezCantalejo E, Slimani N, Scalbert A, Fedirko V, Hjartaker A, Engeset D, Skeie G, Boeing H, Forster J, Li K, Teucher B, Agnoli C, Tumino R, Mattiello A, Saieva C, Johansson I, Stenling R, Redondo ML, Wallstrom P, Ericson U, Khaw KT, Mulligan AA, Trichopoulou A, Dilis V, Katsoulis M, Peeters PH, Igali L, Tjonneland A, Halkjaer J, Touillaud M, Perquier F, Fagherazzi G, Amiano P, Ardanaz E, Bredsdorff L, Overvad K, Ricceri F, Riboli E, Gonzalez CA (2012) Dietary flavonoid and lignan intake and gastric adenocarcinoma risk in the European Prospective Investigation into Cancer and Nutrition (EPIC) study. Am J Clin Nutr 96(6): 1398-1408.

This work is published under the standard license to publish agreement. After 12 months the work will become freely available and the license terms will switch to a Creative Commons AttributionNonCommercial-Share Alike 4.0 Unported License.

Supplementary Information accompanies this paper on British Journal of Cancer website (http://www.nature.com/bjc) 